\title{
Análise de escores preditores de mortalidade após o primeiro ano de internação para pacientes cirróticos descompensados
}

\author{
Analysis of predictive scores for mortality after the first year of hospitalization for decompensated \\ cirrhotic patients
}

Análisis de puntuaciones predictivas de mortalidad después del primer año de hospitalización de pacientes cirróticos descompensados

Recebido: 15/11/2021 | Revisado: 24/11/2021 | Aceito: 28/11/2021 | Publicado: 09/12/2021

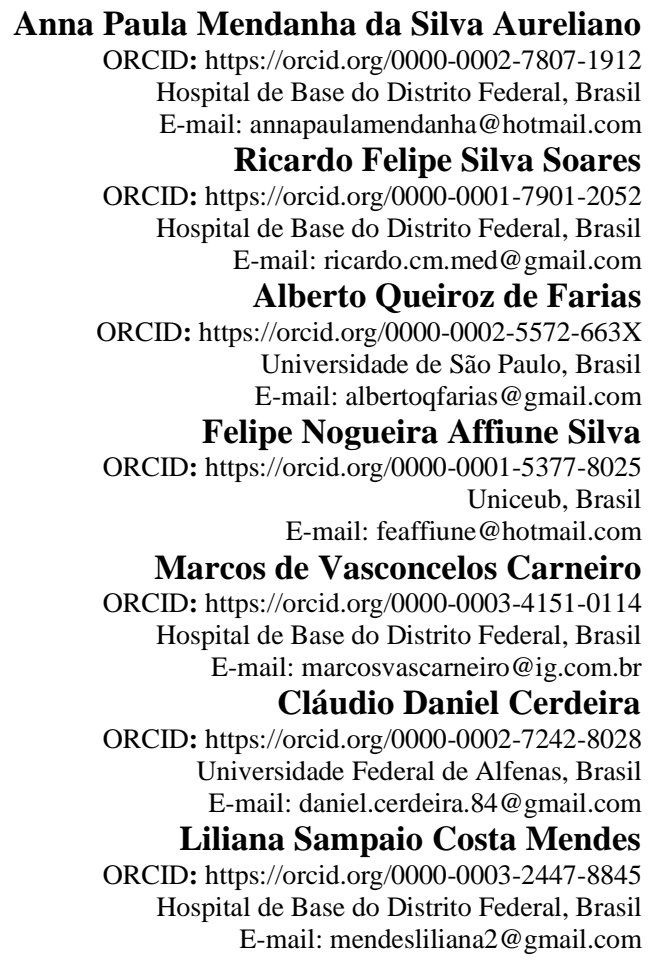

\begin{abstract}
Resumo
Pacientes com cirrose hepática podem evoluir para quadros de descompensação, refletindo em altas taxas de mortalidade. Escores preditores de prognóstico e mortalidade são ferramentas uteis no manejo do paciente com doença hepática crônica. O presente estudo investigou o potencial de escores e modelos preditores de mortalidade após o primeiro ano de internação em pacientes cirróticos atendidos em um hospital terciário do Distrito Federal, Brasil. Este é um estudo de coorte prospectiva que incluiu indivíduos adultos portadores de cirrose hepática, admitidos por descompensação aguda da cirrose, entre novembro de 2018 e maio de 2021 . Os critérios para o término da prospecção foi um dos seguintes desfechos: fim do período supracitado, óbito ou transplante. Foram considerados para este estudo pacientes dos sexos masculino e feminino com cirrose admitidos no hospital por mais de um dia para tratamento da descompensação aguda, e inclusos após enquadramento nos critérios do estudo. As seguintes variáveis foram analisadas: sexo, faixa etária, etnia, causa base da cirrose, razão da admissão, presença de ascite, presença de encefalopatia, valores de exames em admissão (creatinina, sódio, bilirrubina total, INR, albumina) e gravidade de doença hepática pelos modelos de sistemas de escores CLIF-SOFA, MELD, MELD-Na e Child-Pugh, aferidos na admissão no intuito de prever mortalidade após um ano de seguimento. Dos 115 pacientes com cirrose hepática inicialmente inclusos, 63 foram excluídos do estudo por câncer ao longo do follow up, portanto, 52 completaram, sendo $65 \%$ do sexo masculino. Hepatite alcoólica foi a principal causa base da cirrose (46\%), e sangramento intestinal principal causa de internação na descompensação (35\%). Entre os parâmetros bioquímicos isolados, apenas albumina à admissão associou-se significativamente com o desfecho mortalidade em um ano. Entre os modelos avaliados, o CLIF-SOFA apresentou significativa capacidade como preditor relacionado a mortalidade neste período.

Palavras-chave: Cirrose descompensada; Mortalidade; Albumina; CLIF-SOFA.
\end{abstract}




\begin{abstract}
Patients with liver cirrhosis can progress to decompensation, reflecting in high mortality rates. Scores predictors of prognosis and mortality are useful tools in the management of patients with liver disease. The present study investigated the potential of scores and models to predict mortality after the first year of hospitalization in cirrhotic patients treated at a tertiary hospital in the Federal District, Brazil. This is a prospective cohort study that included adult individuals with liver cirrhosis, admitted for acute decompensation of cirrhosis, between November 2018 and May 2021. The criteria for the end of the prospection was one of the following outcomes: end of the period above, death or transplantation. Patients (male and female) with cirrhosis admitted at the hospital for more than one day for treatment of acute decompensation were included according to the previous criteria of the study. The following variables were analyzed: sex, age group, ethnicity, underlying cause of cirrhosis, reason for admission, presence of ascites, presence of encephalopathy, values of biochemical tests on admission (creatinine, sodium, total bilirubin, INR, albumin) and severity of liver disease by CLIF-SOFA, MELD, MELD-Na and Child-Pugh scoring system models, measured in the admission to predict mortality after one year. Of the 115 patients with liver cirrhosis initially included, 63 were excluded for cancer during follow-up, therefore, 52 completed the study, 65\% males. Alcoholic hepatitis was the main underlying cause of cirrhosis (46\%), and intestinal bleeding was the main cause of hospitalization in decompensation (35\%). Among the isolated biochemical parameters, only albumin on admission was significantly associated with the mortality outcome in one year. Among the models evaluated, the CLIF-SOFA showed a significant capacity as a predictor related to mortality in this period.
\end{abstract}

Keywords: Decompensated cirrhosis; Mortality; Albumin; CLIF-SOFA.

\title{
Resumen
}

Los pacientes con cirrosis hepática pueden progresar a descompensación, lo que refleja altas tasas de mortalidad. Las puntuaciones predictoras de pronóstico y mortalidad son herramientas útiles en el manejo de pacientes con enfermedad hepática. El presente estudio investigó el potencial de puntajes y modelos para predecir la mortalidad después del primer año de hospitalización en pacientes cirróticos atendidos en un hospital terciario del Distrito Federal, Brasil. Se trata de un estudio de cohorte prospectivo que incluyó individuos adultos con cirrosis hepática, ingresados por descompensación aguda de cirrosis, entre noviembre de 2018 y mayo de 2021. El criterio para el fin de la prospección fue uno de los siguientes desenlaces: fin del período mencionado, muerte o trasplante. Se consideraron todos los pacientes (hombres y mujeres) con cirrosis ingresados en el hospital durante más de un día para el tratamiento de la descompensación, incluidos seguindo los criterios do estudo. Se analizaron las siguientes variables: sexo, grupo de edad, etnia, causa subyacente de la cirrosis, motivo de ingreso, presencia de ascitis, presencia de encefalopatía, valores de las pruebas al ingreso (creatinina, sodio, bilirrubina total, INR, albúmina) y gravedad de la enfermedad hepática mediante los modelos del sistema de puntuación CLIF-SOFA, MELD, MELD-Na y Child-Pugh, medidos al ingreso para predecir mortalidad después de um año de seguimiento. De los 115 pacientes con cirrosis hepática incluidos inicialmente, 63 fueron excluidos por cáncer durante el seguimiento, por lo que 52 completaron el estudio, 65\% hombres. La hepatitis alcohólica fue la principal causa subyacente de cirrosis (46\%) y el sangrado intestinal fue la principal causa de hospitalización en descompensación (35\%). Entre los parámetros bioquímicos aislados, solo la albúmina al ingreso se asoció significativamente con el resultado de la mortalidad. Entre los modelos evaluados, el CLIF-SOFA mostró una capacidad significativa como predictor relacionado con la mortalidad en este período.

Palabras clave: Cirrosis descompensada; Mortalidad; Albúmina; CLIF-SOFÁ.

\section{Introdução}

A cirrose é um evento histopatológico definido pela substituição difusa do tecido hepático normal por nódulos de estrutura alterada e tecido fibroso de cicatrização, sendo o estágio mais avançado do curso clínico de certas doenças hepáticas crônicas (Lida et al., 2005; Shah et al., 2015; Bernardi et al., 2020). Entre as causas mais comum do desfecho cirrótico, encontram-se as doenças crônicas como as hepatites virais (como as Hepatites B e C) ou autoimunes e doenças metabólicas, vasculares ou biliares, definidas como sendo as doenças de base do evento cirrótico, sendo também a hepatite por alcoolismo crônico uma das principais causas (Lida et al., 2005; Jeong et al., 2016). Em pacientes cirróticos, a presença de ascite, hemorragia varicosa, doença infecciosa, encefalopatia hepática, ou uma combinação destes fatores, define o início de um episódio de descompensação hepática, sendo que tal transição para a descompensação pode ser devido a progressão da doença de base ou um insulto agudo grave do fígado, com repetidos quadros de descompensação aguda (DA) da cirrose podendo ocorrer (Mansour \& McPherson, 2018; Bernardi et al., 2020; Xu et al., 2021). A descompensação nestes casos é uma condição grave, associada a maior tempo de hospitalização e uma mais elevada taxa de mortalidade, $\sim 20 \%$ vs. $\sim 7 \%$ da cirrose 
compensada ( 10-20\% no próprio hospital), elevando a já alta taxa de mortalidade relacionada a cirrose (Samonakis et al., 2014; Harrison, 2015; Mansour \& McPherson, 2018).

É cada vez mais estudado que os pacientes dificilmente morrem como consequência de uma destruição irreversível hepática. Na verdade, a maioria das causas de mortes de pacientes hepatopatas é uma deterioração aguda promovida por um evento precipitante, sendo denominada como insuficiência hepática aguda sobre crônica (Acute-on-Chronic Liver Failure ACLF) (Moreuau, 2013). Os critérios diagnósticos do ACLF definidos pelo EASL-CLIF foram fundamentados em três dados e uma avaliação criteriosa das características clínicas dos pacientes. Um dos dados mais importantes considerados é a existência de insuficiência orgânica. O escore de falência orgânica sequencial e insuficiência crônica hepática (CLIF-SOFA, do inglês Chronic Liver Failure-Sequential Organ Failure Assessment) permite a avaliação hepática, renal, cerebral, da coagulação e da respiração por um sistema de escores que define seis subcategorias. Baseado nesses critérios, os pacientes portadores de cirrose hepática descompensada são estratificados em quatro grupos de gravidade. (Jalan et al., 2014).

Em pacientes com cirrose, a retenção renal de sódio ocorre em $60 \%$ dos pacientes, levando a ascite ou edema periférico (Harrison, 2015). Normalmente, a ascite é a primeira manifestação dos episódios de descompensação, surgindo em $\sim 50 \%$ dos pacientes com cirrose em acompanhamento de 10 anos, sendo associado a 50\% de mortalidade em dois anos após detecção (Harrison, 2015). Outra condição associada a descompensação é o envolvimento do sistema nervoso central (SNC) com o surgimento da encefalopatia hepática, sendo uma disfunção do cérebro causada por disfunção hepática e se manifestando com uma variada gama de alterações neurológicas e/ou psiquiátricas (Harrison, 2015). O desenvolvimento da encefalopatia hepática é associado a uma alta taxa de mortalidade dos pacientes cirróticos em 5 anos, alcançando até $65 \%$ (Harrison, 2015).

Estas alterações patológicas em sistemas e marcadores de funções orgânicas: hepática (bilirrubina, albumina, protrombina, entre outros) e renal (creatinina), são componentes de alguns do escores usados para estabelecer uma classificação/estratificação da doença hepática. Alguns modelos ou sistemas de escores que englobam valores preditores de prognostico e mortalidade tem sido propostos em pacientes com doença hepática crônica, muito utilizados em pacientes com cirrose descompensada, devido aos danos hepáticos e extra-hepáticos (rins, sistema cardiovascular e SNC) nesta condição (Harrison, 2015, Wu et al., 2018; Xiao et al., 2021). Dentre estes, destacam-se os critérios Child-Pugh, MELD (do inglês Model for End-Stage Liver Disease) e CLIF-SOFA, com grande significado clínico, uma vez que tais modelos preditores podem em última análise predizer readmissão, tempo de hospitalização, morbimortalidade, além de orientar as melhores opções terapêuticas do paciente (Jeong et al., 2016; Wu et al., 2018; Xiao et al., 2021; Xu et al., 2021). Os componentes do Child-Pugh são presença/graus de encefalopatia e ascite, tempo de protrombina ou índice internacional normalizado (INR) para avaliar sangramentos, albumina e bilirrubina séricas; com pontuação servindo como classificação funcional para avaliar sobrevivência entre 1-2 anos. O MELD utiliza como componentes os valores séricos de bilirrubina e creatinina e o INR; sendo mais utilizado para sobrevivência a curto-médio prazo (3 meses). O CLIF-SOFA utiliza os parâmetros respiratórios/pulmonares $\left(\mathrm{PaO}_{2}, \mathrm{SaO}_{2}\right)$, de coagulação (número de plaquetas), do fígado (níveis séricos de bilirrubina), cardiovascular (pressão arterial), do SNC (grau de encefalopatia hepática) e renal (níveis séricos de creatinina), sendo este sistema inicialmente desenvolvido para se classificar a descompensação aguda da cirrose (Jeong et al., 2016).

Em pacientes com cirrose descompensada, o transplante ortotópico de fígado é o tratamento de escolha, sendo que todos os pacientes com diagnóstico de cirrose descompensada devem ser considerados candidatos em potencial para o transplante (Harrison, 2015). Neste contexto, o presente estudo investigou o potencial de escores preditores de mortalidade observados na admissão por descompensação da cirrose, em prever a mortalidade no primeiro ano após a admissão. 


\section{Metodologia}

Trata-se de um estudo de coorte prospectiva que incluiu indivíduos adultos portadores de cirrose hepática, admitidos por descompensação aguda da cirrose em um hospital terciário do Distrito Federal, Brasil, entre novembro de 2018 e maio de 2021. Os critérios para o término da prospecção durante o follow up neste período foi um dos seguintes desfechos: fim do período supracitado, óbito ou paciente transplantado. Foram considerados todos os pacientes (sexos masculino e feminino) com cirrose hepática admitidos no hospital por mais de um dia para tratamento da descompensação aguda de cirrose conforme definido por uma ou mais das seguintes complicações: ascite; encefalopatia hepática; sangramento gastrointestinal; infecções bacterianas ou fúngicas. Os critérios de exclusão foram: gravidez, idade menor que 18 anos, insuficiência hepática aguda ou subaguda sem cirrose subjacente, neoplasia localmente avançada ou metastática atual, doenças extra hepáticas graves conhecidas anteriormente (insuficiência renal crônica dialítica, doença cardíaca grave, doença pulmonar crônica grave e distúrbios psiquiátricos), infecção por HIV, transplante prévio de fígado e pacientes que não podem fornecer consentimento informado prévio. As seguintes variáveis foram analisadas: sexo, faixa etária, etnia, causa base da cirrose, razão da admissão, presença de ascite, presença de encefalopatia, valores de exames de bioquímica em admissão (creatinina, sódio, bilirrubina total, INR, albumina) e, gravidade de doença hepática pelos modelos de escores CLIF-SOFA, MELD, MELD-Na e ChildPugh, sendo estes preditores usados para avaliar os óbitos no primeiro ano do follow up da internação/admissão por descompensação dos pacientes. As pontuações dos escores CLIF-SOFA, MELD, MELD-Na e Child-Pugh foram obtidas de acordo com os critérios previamente reportados (Jeong et al., 2016), sendo o valor médio na amostra calculado. Após coleta, os dados foram tabulados utilizando-se o pacote Microsoft Office Excel® 2013. As análises estatísticas foram realizadas no BioEstat versão 5.3. A normalidade da distribuição das variáveis foi avaliada pelo teste de Kolmogorov-Smirnov. A comparação entre médias em variáveis contínuas com distribuição normal foi realizada através do teste t de Student, o qual foi substituído pelo teste de Mann-Whitney em casos de variâncias desiguais (dados sem distribuição normal). A associação entre variáveis categóricas (qualitativas) foi analisada através do teste de Qui-quadrado $\left(\chi_{2}\right)$. Para os dados demográficos da Tabela 2, usou-se o Teste G com correção de Williams. A performance dos escores em predizer a mortalidade a curto, médio e longo prazo ( $\geq 1$ ano) foi analisada pelo cálculo da área sob a curva ROC (receiver operating characteristics). Baseado nas curvas ROC, os melhores pontos de corte foram escolhidos e a partir de então caracterizada sensibilidade, especificidade, valor preditivo positivo, valor preditivo negativo e acurácia.

Este estudo foi aprovado pelo comitê local de ética em pesquisa envolvendo seres humanos, sob CAAE 81120117.4.2005.8153 e parecer de aprovação número 2.865.350. Os pacientes voluntários foram plenamente informados sobre todos os aspectos do estudo e de sua participação, além de fornecerem consentimento formal através do esclarecimento e assinatura do TCLE, tudo em absoluta consonância com os preceitos da resolução n. 466/12 do CNS.

\section{Resultados}

A partir de 115 pacientes com cirrose hepática internados no período, 63 foram excluídos por câncer - carcinoma hepatocelular ou colangiocarcinoma - portanto, 52 foram incluídos e completaram o presente estudo. A Tabela 1 apresenta as variáveis demográficas relevantes dos 52 pacientes, bem como os dados gerais de saúde relacionados a admissão e causa base da cirrose descompensada. Houve maior prevalência da descompensação tendo como causa base a hepatite por álcool (24 dos 52 pacientes), e a etiologia viral (6 casos) da cirrose foi significativamente associada ao grupo óbito ( $\mathrm{p}=0,002)$, comparada as outras causas. A Tabela 2 é descritiva e traz informações dos pacientes internados por descompensação cirrótica. 
Tabela 1 - Perfil demográfico e de saúde de pacientes cirróticos descompensados hospitalizados. Brasília, 2021

\begin{tabular}{|c|c|c|c|c|c|c|}
\hline \multirow[t]{3}{*}{ VARIÁVEL } & & \multicolumn{4}{|c|}{ ÓBITOS } & \multirow{3}{*}{$p$ valor } \\
\hline & & \multicolumn{2}{|c|}{$\operatorname{Sim}(n=23)$} & \multicolumn{2}{|c|}{ Não $(n=29)$} & \\
\hline & & $\mathrm{n}$ & $\%$ & $\mathrm{n}$ & $\%$ & \\
\hline Faixa etária & $>40$ & 21 & 91 & 25 & 86 & \\
\hline (anos) & $<40$ & 2 & 9 & 4 & 14 & 0,89 \\
\hline \multirow[t]{3}{*}{ Etnia } & Branco & 12 & 52 & 15 & 52 & \\
\hline & Negro & 10 & 44 & 13 & 45 & 0,986 \\
\hline & Indígena & 1 & 4 & 1 & 3 & \\
\hline \multirow[t]{4}{*}{ Causa base/Etiologia } & Cirrose alcoólica & 11 & 48 & 13 & 45 & \\
\hline & Cirrose por $\mathrm{NASH}^{1}$ & 5 & 22 & 7 & 24 & $0,002 *$ \\
\hline & Cirrose causada por vírus ${ }^{2}$ & 6 & 26 & 0 & 0 & \\
\hline & Outras & 1 & 4 & 9 & 31 & \\
\hline \multirow[t]{5}{*}{ Razão da admissão ${ }^{3}$} & Sangramento gastrointestinal & 9 & 39 & 9 & 31 & \\
\hline & Ascite & 4 & 17 & 5 & 17 & \\
\hline & Encefalopatia & 4 & 17 & 2 & 7 & 0,564 \\
\hline & Outras infecções & 5 & 22 & 9 & 31 & \\
\hline & Outras causas & 1 & 4 & 4 & 14 & \\
\hline
\end{tabular}

${ }^{1} \mathrm{NASH}$ : Doença hepática gordurosa não alcoólica. ${ }^{2} \mathrm{HCV}, \mathrm{HBV}, \mathrm{HBS} .{ }^{3}$ na admissão. O perfil destes eventos mudou ao longo do follow up, por exemplo, presença de infecções, sangramento, encefalopatia e ascite. *Significativo de acordo com o Teste $\mathrm{G}$ com correção de Williams. Fonte: Autores.

Tabela 2 - Associação de variáveis categóricas em admissão e desfecho óbito dentro de um ano em pacientes cirróticos descompensados hospitalizados. Brasília, 2021

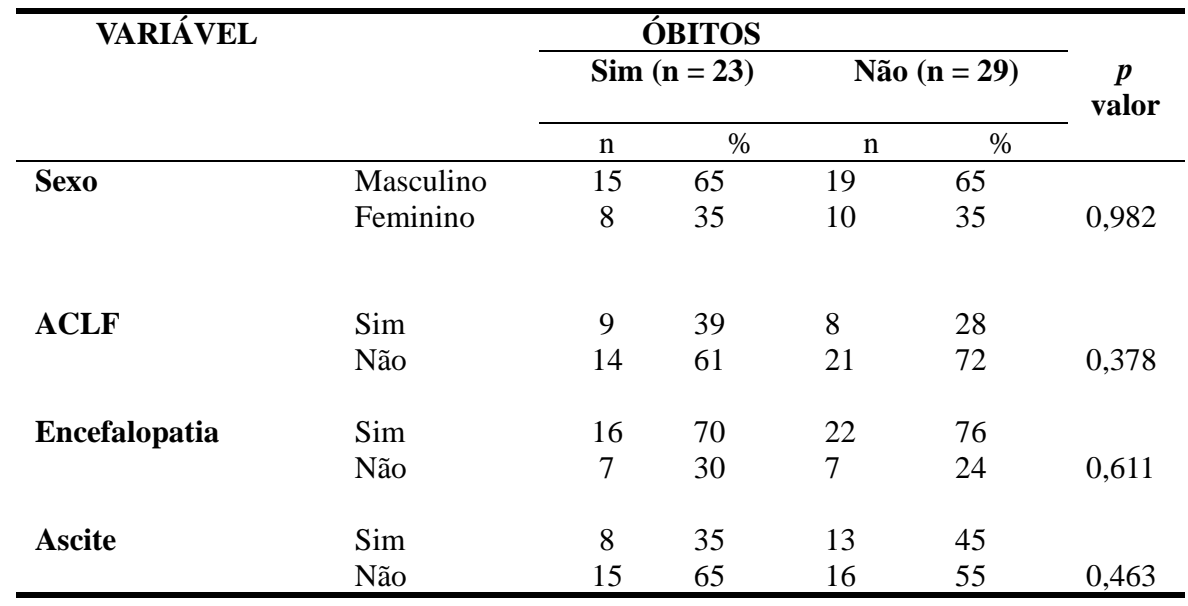

Nota: considerou-se no grupo ACLF, pacientes que apresentavam a síndrome na admissão ou desenvolveram durante a mesma internação. $p$ valor para $\chi^{2}$. Fonte: Autores.

Como observado na Tabela 3, os menores níveis de albumina à admissão associaram-se significativamente $(\mathrm{p}<0,05)$ com o desfecho óbito. Os outros parâmetros bioquímicos não se relacionaram com o desfecho óbito. 
Tabela 3 - Variáveis laboratoriais de admissão associadas a desfecho óbito dentro de um ano em pacientes cirróticos descompensados hospitalizados. Brasília, 2021.

\begin{tabular}{|c|c|c|c|}
\hline \multirow{3}{*}{ VARIÁVEL } & $\overline{\text { ÓB }}$ & & \multirow{3}{*}{$p$ valor } \\
\hline & Sim $(n=23)$ & Não $(n=29)$ & \\
\hline & \multicolumn{2}{|c|}{ Média \pm DP } & \\
\hline Creatinina (mg/dL) & $1,47 \pm 1,15$ & $1,16 \pm 0,51$ & $0,712(\#)$ \\
\hline Sódio $(\mathbf{m E q} / \mathbf{L})$ & $137,4 \pm 4,86$ & $135,9 \pm 5,72$ & 0,33 \\
\hline Bilurrubina total & $5,04 \pm 5,5$ & $4,43 \pm 6,14$ & 0,710 \\
\hline INR & $1,83 \pm 0,71$ & $1,71 \pm 0,62$ & 0,542 \\
\hline Albumina (g/dL) & $2,53 \pm 1,01$ & $3,1 \pm 0,81$ & $0,02(*)$ \\
\hline
\end{tabular}

Nota: Variáveis contínuas expressas em média \pm desvio padrão (DP). (\#) p valor para teste de MannWhitney (prova não paramétrica para amostras desiguais), nos demais casos $p$ valor de Teste t de student. $(*)$ denota diferença estatisticamente significativa entre grupos $(p<0,05)$. Fonte: Autores.

Conforme a Tabela 4, apenas o CLIF-SOFA relacionou-se significativamente $(\mathrm{p}<0,05)$ com o desfecho óbito. Na Tabela 5 observa-se que o MELD e Child-Pugh apresentam acurácia semelhantes.

Tabela 4 - Comparação de escores segundo desfecho óbito no primeiro ano após a admissão em pacientes cirróticos descompensados hospitalizados. Brasília, 2021

\begin{tabular}{|c|c|c|c|}
\hline \multirow{3}{*}{ VARIÁVEL } & & & \multirow{3}{*}{$p$ valor } \\
\hline & $\operatorname{Sim}(n=23)$ & Não $(n=29)$ & \\
\hline & \multicolumn{2}{|c|}{ Média \pm DP } & \\
\hline MELD & $19,74 \pm 7,64$ & $17 \pm 7,6$ & 0,2 \\
\hline MELD-Na & $21,3 \pm 7,6$ & $20,1 \pm 7,56$ & 0,59 \\
\hline Child-Pugh & $9,1 \pm 2,47$ & $8,5 \pm 2,89$ & 0,477 \\
\hline CLIF-SOFA & $5,78 \pm 2,87$ & $4,48 \pm 1,19$ & $0,04 *$ \\
\hline
\end{tabular}

Nota: Valores expressos em média \pm desvio padrão (DP). $p$ valor para Teste $\mathrm{t}$ de student. * denota diferença estatisticamente significativa entre grupos $(p<0,05)$. MELD = Model for End-Stage Liver Disease; CLIF-SOFA = Chronic Liver Failure- Sequential Organ Failure Assessment. Fonte: Autores.

Tabela 5 - Desempenho dos diferentes modelos prognósticos na predição de mortalidade em um ano para os pacientes cirróticos descompensados avaliados neste estudo, definidos por curvas ROC. Brasília, 2021.

\begin{tabular}{lc|c|c|c|c|c}
\hline ESCORE & PC & S (\%) & E (\%) & \multicolumn{2}{c}{ VPP (\%) $(\%)$} & ACURÁCIA \\
MELD & $\geq 16$ & 78 & 52 & 56 & 75 & 63 \\
MELD-Na & $\geq 22$ & 52 & 59 & 50 & 61 & 56 \\
\hline Child-Pugh & $\geq 9$ & 65 & 62 & 58 & 69 & 63 \\
\hline CLIF-SOFA & $\geq 5$ & 60 & 55 & 52 & 64 & 58 \\
\hline
\end{tabular}

Nota: $\mathrm{PC}=$ ponto de corte; $\mathrm{S}=$ sensibilidade; $\mathrm{E}$ = especificidade; $\mathrm{VPP}=$ valor preditivo positivo; $\mathrm{VPN}$ = valor preditivo negativo; $\mathrm{MELD}=$ Model for End-Stage Liver Disease; CLIF-SOFA = Chronic Liver Failure- Sequential Organ Failure Assessment; ROC = Receiver Operating Characteristic curve. Fonte: Autores. 


\section{Discussão}

No presente estudo avaliou-se uma amostra de 52 pacientes atendidos em um hospital terciário, internados por descompensação da cirrose hepática. Dados demográficos permitiu a caracterização da amostra, com semelhante distribuição entre pacientes brancos e negros, faixa etária predominantemente $>40$ anos, e maior percentual para o sexo masculino. Tais dados são importantes, uma vez que fatores epidemiológicos relacionados a específicas populações influenciam a incidência e os desfechos associados a cirrose hepática, como idade acima de 40 anos que determinam, em geral, maior período de danos e maior vulnerabilidade as complicações. No presente estudo, a doença alcoólica predominou como causa base da cirrose nos pacientes cirróticos descompensados e, houve associação significativa entre a causa base sendo hepatite viral e o desfecho óbito ao primeiro ano. Estudos já têm demonstrado que mesmo após o controle ou erradicação de vírus das Hepatites B e C, os portadores de cirrose persistem sobre risco de carcinoma hepatocelular (Trevisani et al., 2007; El-Serag, 2012), sendo que a Hepatite $\mathrm{C}$ dominou até recentemente as listas de transplante hepático no mundo, uma vez que a cirrose e suas complicações relacionadas podem desencadear desfechos de óbito, também ocorrendo ascite com mais frequência em pacientes cirróticos devido a hepatites virais (Trevisani et al., 2007; Samonakis et al., 2014). A etiologia álcool, em longo prazo, tem uma chance maior de recuperação de inflamação; isso explicaria o desfecho óbito estar associado a outras causas de hepatopatia, como a hepatite viral no presente e em outros estudos (Trevisani et al., 2007; Toshikuni et al., 2009; El-Serag, 2012). Entre as principais causas de admissão/internação na descompensação observadas no presente estudo, destacam-se o sangramento gastrointestinal e a ascite, corroborando a literatura (Martins et al., 2013; Harrison, 2015). Xu et al. (2021), em um estudo de coorte na China, observaram que a ascite foi a principal causa de admissão em casos de descompensação.

No presente estudo, as dosagens de sódio sérico, creatinina e os valores de INR (que avalia coagulação) não foram capazes de se correlacionar com o óbito ao primeiro ano, embora a creatinina, o sódio sérico e INR sejam empregados em escores MELD e MELD-Na. Esses escores se correlacionam mais com a predição de mortalidade dentro de 3 meses (Xiao et al., 2021; Xu et al., 2021). Apenas albumina, isoladamente, mostrou-se um escore preditor significativo para mortalidade dentro de um ano após internação por descompensação. Bernardi et al. (2020) também observaram a relevância deste marcador bioquímico na predição de alguns desfechos relacionados a cirrose hepática descompensada, como óbito. Neste contexto, as concentrações plasmáticas desta proteína relacionam-se a uma melhora na volemia e, mudanças estruturas dela são observadas na fase aguda da descompensação, comprometendo sua funcionalidade. Os autores citam o papel pleiotrópico da albumina em contextos fisiológicos e na manutenção da saúde frete as agressões, e a aplicabilidade como um potencial novo alvo terapêutico para o tratamento da cirrose descompensada. Esse papel protetor da albumina também é corroborado no presente estudo, onde mais elevados níveis desta proteína associou-se positivamente a sobrevivência. Ainda, a administração a longo prazo de albumina em pacientes cirróticos descompensados pode reduzir as complicações, facilitar o manejo da ascite, reduzir hospitalizações (readmissões) e melhorar a taxa de sobrevivência. Contudo, mais estudos para compreender a melhor dosagem a ser administrada e o contexto ideal devem ser conduzidos (Bernardi et al., 2020). Considerando outras condições clínicas, recentemente, a albumina sérica foi apontada como um escore preditor independente também em pacientes oncológicos (Lima et al., 2021). A albumina faz parte do Child-Pugh, que tradicionalmente estratifica portadores de cirrose ao longo dos anos, sendo este apenas um dos itens do critério, que também pode se modificar ao longo do acompanhamento, de acordo com intercorrências. De acordo com Calmet et al. (2019), alterações nutricionais podem ocorrer por conta da cirrose, aumentando em frequência os mais altos escores Child-Pugh e elevando as taxas de morbimortalidade nestes pacientes. Além disso, conforme observado no presente estudo, com uma casuística composta de mais pacientes cirróticos alcoolistas, mais recentemente, a desnutrição foi descrita como um fator de impacto na mortalidade de cirróticos (guideline Nutrition EASl 2018) e, é possível que o estado nutricional seja relacionado com a hipoalbuminemia e, consequentemente, com o desfecho óbito a longo prazo (Calmet et al., 2019). Corroborando isto, Martins et al. (2013) observaram em seu estudo de revisão que 
pacientes cirróticos, principalmente alcoolistas, desenvolvem um quadro de desnutrição proteico-energética (DPE), dependendo do grau de envolvimento relacionado a absorção e/ou metabolismo hepático. Estas alterações afetam principalmente os níveis normais de albumina, a principal proteína sérica, uma vez que tanto a absorção de aminoácidos quanto a biossíntese nos hepatócitos são comprometidas (Martins et al. 2013; Bernardi et al., 2020).

Os diferentes modelos preditores são 'propostos para se avaliar o impacto da descompensação da cirrose e outras doenças hepáticas crônicas e terminais, no que diz respeito ao prognostico que reflete na readmissão e sobrevivência de pacientes (Jeong et al., 2016), sendo estes modelos para mortalidade aqui avaliados contando os óbitos em até um ano de internação. Assim como para mortalidade, Xu et al. (2021) observaram que os modelos MELD e MELD-Na, apesar de predizer mortalidade a curto/médio prazo ( 3 meses), apresentaram menor acurácia em predizer os fatores de risco para readmissão (principalmente por infecções), comparado a um critério padronizado chamado nomograma, tendo como base os primeiros 90 dias de internação. Devido a priorização energética comprometendo a resposta imune e uma vez que as doenças infecciosas constituem uma considerável causa de comorbidade e mortalidade em pacientes cirróticos descompensados ou com doença hepática crônica terminal, sendo também um fator precipitador da descompensação aguda (de Mattos et al., 2003; Bernardi et al., 2020), Xiao et al. (2021), observaram que na admissão o modelo MELD apresenta melhor predição dos impactos da gravidade na infecção pelo SARS-CoV-2 na COVID-19 (em curto prazo) em pacientes cirróticos com descompensação, enquanto que o Child-Pugh relacionou-se a não sobrevivência entre os pacientes (a médio/longo prazo).

Levando-se em conta a principal (mais prevalente) causa base de cirrose observada neste estudo, que também é relatada como uma das principais causas de descompensação (Samonakis et al., 2014), a cirrose hepática desenvolvida pelo alcoolismo crônico, o modelo CLIF-SOFA mostrou-se um significativo preditor com considerável acurácia para avaliar o impacto da doença sobre mortalidade tardia (dentro de um ano). Jeong et al. (2016) observaram que o CLIF-SOFA é um considerável modelo preditor também no prognóstico para avaliar mortalidade a curto prazo de internação (até 30 dias) em pacientes com cirrose descompensada em fase aguda tendo como causa base o alcoolismo crônico (induzida pelo consumo crônico de álcool, >60g/dia para homens e >40g/dias para mulheres), na presença de encefalopatia hepática.

\section{Conclusão}

O modelo de escore CLIF-SOFA apresentou significativa capacidade para predição de mortalidade tardia dentro de um ano em pacientes cirróticos de várias etiologias, admitidos por descompensação da cirrose. Na referida amostra, os demais escores MELD, MELD-Na e Child-Pugh não estiveram relacionados à maior mortalidade em um ano. São necessários mais estudos para corroborar estes achados.

\section{Referências}

Bernardi, M., et al. (2020). Albumin in decompensated cirrhosis: new concepts and perspectives. Gut, 69, 1127.

Calmet, F., et al. (2019). Nutrition in patients with cirrhosis. Gastroenterology \& Hepatology, 15, 248.

de Mattos, A. A., et al. (2003). Infecção bacteriana no paciente cirrótico. Arq Gastroenterol, 40, 11.

El-Serag, H. B. (2012). Epidemiology of viral hepatitis and hepatocellular carcinoma. Gastroenterology, $142,1264$.

Harrison, P. M. (2015). Management of patients with decompensated cirrhosis. Clinical Medicine, 15(2), 201.

Jalan, R., et al. (2014). Development and validation of a prognostic score to predict mortality in patients with acute-on- chronic liver failure. $J$ Hepatol, 61 . $1038-47$.

Jeong, J. H., et al. (2016). CLIF-SOFA score and SIRS are independent prognostic factors in patients with hepatic encephalopathy due to alcoholic liver cirrhosis. Medicine, 95, 26.

Lida, V. H., et al. (2005). Cirrose hepática: aspectos morfológicos relacionados às suas possíveis complicações. J Bras Patol Med Lab, 41(1), 29. 
Research, Society and Development, v. 10, n. 16, e159101623315, 2021

(CC BY 4.0) | ISSN 2525-3409 | DOI: http://dx.doi.org/10.33448/rsd-v10i16.23315

Lima, E. N. S., et al. (2021). Serum albumin is independent predictor of hospital mortality in patients with cancer. Revista Brasileira de Cancerologia, 67(4), e-071209.

Mansour, D., \& McPherson, S. (2018). Management of decompensated cirrhosis. Clinical Medicine, 18(2), s60.

Martins, F. L. (2013). Nutrição em paciente cirrótico. HU Revista, 39, 45

Moreau, R., et al. (2013). Acute-on- chronic liver failure is a distinct syndrome developing in patients with acute decompensation of cirrhosis. Gastroenterology, 144, 1426- 37 .

Samonakis, D. N., et al. (2014). Clinical outcomes of compensated and decompensated cirrhosis: A long term study. World J Hepatol, 6(7), 504.

Shah, N. L., et al. (2015). Management options in decompensated cirrhosis. Hepatic Medicine: Evidence and Research, 7, 43.

Trevisani, F., et al. (2007). Impact of etiology of cirrhosis on the survival of patients diagnosed with hepatocellular carcinoma during surveillance. Am $J$ Gastroenterol; 102, 1022.

Toshikuni, N., et al. (2009). Comparison of outcomes between patients with alcoholic cirrhosis and those with hepatitis C virus-related cirrhosis. $J$ Gastroenterol Hepatol, 24, 1276.

Xiao, Y., et al. (2021). High Child-Pugh and CRUB65 scores predict mortality of decompensated cirrhosis patients with COVID-19: A 23-center, retrospective study. Virulence, 12(1), 1199.

Xu, X., et al. (2021). Risk stratification score to predict readmission of patients with acute decompensated cirrhosis within 90 days. Front Med, $8,646875$.

Wu, S-L., et al. (2018). Scoring systems for prediction of mortality in decompensated liver cirrhosis: A meta-analysis of test accuracy. World J Clin Cases, 6(15), 995. 